\title{
The Benefits of Astaxanthin to Improve Pain Relief in Patients with Painful Diabetic Neuropathy: An Open-Label, Randomized Controlled Trial
}

\author{
Rizaldy Taslim Pinzon*, Mary Rose Angelina Budi Harsana \\ Faculty of Medicine, Duta Wacana Christian University, Yogyakarta, Indonesia
}

\begin{abstract}
Treatment of diabetic neuropathy is still carried out by providing symptomatic therapy, which only improves $\pm 50 \%$ of the total symptoms felt by patients, but does not tackle the underlying causes of the disease. Astaxanthin is a potent antioxidant, anti-inflammatory, and anti-diabetic carotenoid that could be an additional treatment option. We aimed to measure the effectiveness of administering astaxanthin as an additional therapy to improve the impact of pain and discomfort experienced daily by diabetes mellitus patients with painful diabetic neuropathy. We conducted a randomized experimental study with an open label design of 36 patients who had been diagnosed with painful diabetic neuropathy. The control group was treated with standard treatment for painful diabetic neuropathy, and the experimental group was given both standard and additional therapy of astaxanthin at a dose of $6 \mathrm{mg}$ once per day. The impact of pain was assessed using the Brief Pain Inventory (BPI) before administering astaxanthin and on the $4^{\text {th }}$ and $8^{\text {th }}$ weeks after administering astaxanthin. The administration of therapy showed a significant improvement in the impact of pain experienced daily by patients on both treatment groups $(\mathrm{p}<0.05)$. However, the mean BPI score of the control and the experimental groups did not differ significantly each week $(\mathrm{p}>0.05)$. There is a significant improvement in the BPI of patients with painful diabetic neuropathy who were given additional treatment (add on) of astaxanthin compared to patients who were only given standard treatment for painful diabetic neuropathy.
\end{abstract}

Keywords: astaxanthin; antioxidant; painful diabetic neuropathy; the impact of pain

*corresponding author

Email:drpinzon17@gmail.com

\section{INTRODUCTION}

Diabetes mellitus is one of the non-communicable diseases with the highest causes of death of about 1.6 million people per year. According to the data stated by Indonesia's Basic Health Research in 2018, there were approximately 16 million of Indonesians who suffered from diabetes, and it is estimated to increase up to about 21.3 million people by the year 2030 (Ministry of Health of the Republic of Indonesia, 2018). Diabetes is a group of chronic metabolic disorders characterized by high blood sugar levels (hyperglycemia) caused by defects in insulin secretion, insulin action, or both due to the damage of pancreatic $\beta$-cells. Mistreatment or late treatment of diabetes may lead to further complications. Microvascular complications such as neuropathy, nephropathy, and retinopathy are the complications that often occur in diabetic patients (Wisse, 2018). Unfortunately, about $75 \%$ of patients may be asymptomatic and therefore fail to recognize the early symptoms of this disease, resulting in further serious complications or even death (American Diabetes Association, 2014).
Painful diabetic neuropathy is one of the complications that often occur in patients with diabetes mellitus $(>50 \%)$ and is caused by disruptions in the metabolic and vascular systems. Symptoms of diabetic neuropathy are prickling and tingling sensation, numbness, pain with burning sensation, and stabbing pain in certain body parts (Javed, Petropoulos, Alam, \& Malik, 2015). It is necessary to provide the appropriate treatment for patients who suffer from painful diabetic neuropathy as it limits their daily activities. The treatment available for these patients is still in the form of symptomatic treatment such as pregabalin, gabapentin, and amitriptyline, thus requiring additional treatment (Kaur \& Pandhi, 2011). Therefore, healthcare professional, specifically neurologists and other general practitioners that often handle neuropathy patients with the history of diabetes need to provide the proper treatments and strict glycemic control for diabetic patients with painful diabetic neuropathy to alleviate the pain and prevent further progression of painful diabetic neuropathy to help patients enhance their quality of life and well-being. 
Astaxanthin, a natural carotenoid that acts as a potent antioxidant with its anti-diabetic and anti-inflammatory properties, can can protect nerve damage and reduce neuroinflammation caused by diabetes. Hence, it can relieve the pain and other neuropathy symptoms felt by patients (Davinelli, Scapagnini, \& Nielsen, 2018). Due to this, additional astaxanthin treatment towards the standard treatment of painful diabetic neuropathy is required. Previous studies rarely discuss the use of astaxanthin in symptom improvement, especially in cases concerning painful diabetic neuropathy (Lin, Wen, $\&$ Tsai, 2018). Previous studies focused more on the use of astaxanthin in treating retinopathy and nephropathy that caused by various diseases. One study by Yeh, et al., (2016) focused more on the use of astaxanthin on treating retinopathy, while another study by Chen, et al., (2020) focused more on the use of astaxanthin in treating retinopathy. Therefore, this study was conducted to examine the benefits of astaxanthin on improving the impact of pain relief patients with painful diabetic neuropathy.

\section{METHODS}

\section{Design}

This study was conducted with an experimental randomized controlled trial, active comparator, and an open-label design.

\section{Subject}

This study was conducted on 36 diabetic patients at Bethesda Hospital Yogyakarta diagnosed with painful diabetic neuropathy based on Diabetic Neuropathy Examination (DNE) and Diabetic Neuropathy Symptom (DNS) who have agreed and signed the given informed consent.

\section{Assessment}

Data retrieval was conducted using secondary data obtained from the Brief Pain Inventory (BPI) questionnaire that patients filled out on their selfassessment sheets, by choosing the level of intensity of the symptoms experienced by the patients on a scale of 0 to 100 . Subjects were divided into two groups. The control group received standard treatment for painful diabetic neuropathy only (gabapentin, pregabalin, or a combination with amitriptyline). The experimental group received both standard and additional astaxanthin treatment at a dose of $6 \mathrm{mg}$ once per day for eight weeks. The effect of both treatment groups on the impact of pain on patient's daily activities was assessed using the BPI by questioning 8 items of questions regarding the symptoms experienced by patients before the administration of treatment, and on the 4th and 8th weeks after the administration of the additional treatment. The assessment aimed to carefully analyze the improvement process of painful diabetic neuropathy symptoms in both groups, which is done by analyzing the mean difference of BPI scores from week 0 until week 8. Data retrieval was collected by an enumerator to avoid biases and obtain valid and reliable data.

\section{Statistical Analysis}

The data analysis was performed using the Mann Whitney test to compare dependent variables or outcomes between two independent treatment groups.

\section{Ethical Approval}

This study has complied with all regulations based on The Council for International Organizations of Medical Sciences (CIOMS) 2016 and has received ethical approval from Bethesda Hospital Yogyakarta's Health Research Ethics Commission with ethical approval number of No. 16/KEPK-RSB/II/21.

\section{RESULTS}

\section{Basic Characteristics of Research Subjects}

Thirty-six research subjects with confirmed painful diabetic neuropathy were divided into two groups. The control and experimental groups. One subject in the control group was dropped out from the study due to death by COVID-19 during the study. The research subjects in both groups consisted of 16 men and 20 women, with an average age of $63.89 \pm 8.013$ years old, as shown in Table 1. The comorbid conditions that existed in most subjects were hypertension, by a record of 23 subjects $(63.8 \%)$ followed by cardiovascular disease in 22 subjects $(61.1 \%)$, and gastrointestinal disease in 4 subjects $(0,11 \%)$. The most widely used medication among the subject research is an antihypertensive agent used by 23 subjects $(63.8 \%)$, followed by antiplatelet agents, vitamin B, and statin drugs by 19 (52.7\%), 14 $(38,8 \%)$, and 8 subjects $(22,2 \%)$ respectively. The mean score of DNE in the control group was $4.00 \pm 0.907$, while in the experimental group was $3.83 \pm 0.857$ with a p-value of 0.735 . On the other hand, the mean score of DNS of the control group was $2.39 \pm 0.916$, while the experimental group was $2.50 \pm 1.04$ with a p-value of 0.736 . DNE and DNS scores in both groups did not differ significantly ( $\mathrm{p}>0.05)$, as illustrated in Table 2 .

All subjects were given standard treatment of $6 \mathrm{mg}$ astaxanthin that was proven to have the most effective dose for patients, including study by Tominaga (2009). Therapy was given with either a single treatment of gabapentin or pregabalin or combined treatment with amitriptyline, as shown in Table $3.100 \mathrm{mg}$ gabapentin was administered to 15 subjects $(83.3 \%)$ in the control group and 17 subjects (94.4\%) in the experimental group, while the prescription of $75 \mathrm{mg}$ pregabalin was given to 2 subjects $(11.1 \%)$ in the control group. Combined 
Table 1. Basic characteristics of research subjects

\begin{tabular}{|c|c|c|c|}
\hline & Experimental Group & Control Group & \multirow{2}{*}{$\mathbf{p}$} \\
\hline & $n(\%)$ & $\mathbf{n}(\%)$ & \\
\hline Age (Mean \pm SD) & $65.28 \pm 7.41$ & $62.50 \pm 8.549$ & 0.294 \\
\hline \multicolumn{4}{|l|}{$\operatorname{Sex}$} \\
\hline Male & $7(38.9)$ & $9(50)$ & \multirow{2}{*}{0.502} \\
\hline Female & $11(61.1)$ & $9(50)$ & \\
\hline \multicolumn{4}{|c|}{ Comorbid Conditions } \\
\hline \multicolumn{4}{|l|}{ Hypertension } \\
\hline Yes & $11(61.1)$ & $12(66.7)$ & \multirow{2}{*}{0.729} \\
\hline No & $7(38.9)$ & $6(33.3)$ & \\
\hline \multicolumn{4}{|c|}{ Cardiovascular Disease } \\
\hline Yes & $11(61.1)$ & $11(61.1)$ & \multirow{2}{*}{1.000} \\
\hline No & $7(38.9)$ & $7(38.9)$ & \\
\hline \multicolumn{4}{|c|}{ Gastrointestinal Disease } \\
\hline Yes & $3(16.7)$ & $1(5.6)$ & \multirow{2}{*}{0.289} \\
\hline No & $15(83.3)$ & $17(94.4)$ & \\
\hline \multicolumn{4}{|l|}{ Comedication } \\
\hline \multicolumn{4}{|c|}{ Antihypertensive } \\
\hline Yes & $11(61.1)$ & $12(66.7)$ & \multirow{2}{*}{0.729} \\
\hline No & $7(38.9)$ & $6(33.3)$ & \\
\hline \multicolumn{4}{|l|}{ Antiplatelet } \\
\hline Yes & $10(55.6)$ & $9(50)$ & \multirow{2}{*}{0.738} \\
\hline No & $8(44.4)$ & $9(50)$ & \\
\hline \multicolumn{4}{|l|}{ Statin } \\
\hline Yes & $4(22.2)$ & $4(22.2)$ & \multirow{2}{*}{1.000} \\
\hline No & $14(77.8)$ & $14(77.8)$ & \\
\hline \multicolumn{4}{|l|}{ Vitamin B } \\
\hline Yes & $8(44.4)$ & $6(33.3)$ & \multirow{2}{*}{0.494} \\
\hline No & $10(55.6)$ & $12(66.7)$ & \\
\hline
\end{tabular}

Data presented as $\mathrm{n}(\%)$ or mean $\pm \mathrm{SD} ; \mathrm{p}$, significance between experimental and control group; Independent T-test for normally distributed data, Mann-Whitney test for abnormally distributed data, Wilcoxon test for comparing the significance between a pair of data. The statistically significant difference is shown as * $(p<0.05)$.

Table 2. Mean score of DNE and DNS in both research groups

\begin{tabular}{llll}
\hline & $\begin{array}{l}\text { Experimental Group } \\
(\mathbf{n = 1 8})\end{array}$ & $\begin{array}{l}\text { Control Group } \\
(\mathbf{n}=\mathbf{1 8})\end{array}$ & $\boldsymbol{p}$ \\
\hline DNE & $3.83 \pm 0.857$ & $4.00 \pm 0.907$ & 0.735 \\
DNS & $2.50 \pm 1.04$ & $2.39 \pm 0.916$ & 0.736 \\
\hline
\end{tabular}

Data presented as n; p, significance between experimental and control group; Independent T-test for normally distributed data, Mann-Whitney test for abnormally distributed data. The statistically significant difference is shown as $*(p<0.05)$. 
Table 3. Standard treatment of both treatment groups

\begin{tabular}{llll}
\hline Standard Treatment & $\begin{array}{l}\text { Experimental Group } \\
\mathbf{n}(\%)\end{array}$ & $\begin{array}{l}\text { Control Group } \\
\mathbf{n}(\%)\end{array}$ & $\boldsymbol{p}$ \\
\hline Gabapentin $100 \mathrm{mg}$ & $17(94.4)$ & $15(83.3)$ & \\
Pregabalin $75 \mathrm{mg}$ & $0(0)$ & $2(11.1)$ & 0.248 \\
$\begin{array}{l}\text { Gabapentin } 100 \mathrm{mg} \\
+ \text { Amitriptyline } 25 \mathrm{mg}\end{array}$ & $0(0)$ & $1(5.6)$ & \\
$\begin{array}{l}\text { Gabapentin } 300 \mathrm{mg} \\
+ \text { Amitriptyline } 25 \mathrm{mg}\end{array}$ & $1(5.6)$ & $0(0)$ & \\
\hline
\end{tabular}

Data presented as $\mathrm{n} ; \mathrm{p}$, significance between experimental and control group; Independent T-test for normally distributed data, Mann-Whitney test for abnormally distributed data. The statistically significant difference is shown as $*(p<0.05)$.

Table 4. Mean BPI score of experimental group and control group

\begin{tabular}{llll}
\hline BPI Score & $\begin{array}{l}\text { Experimental Group } \\
(\mathbf{n}=\mathbf{1 8})\end{array}$ & Control Group (n=17) & $\boldsymbol{p}$ \\
\hline Pain with burning sensation & & & \\
Baseline (Week 0) & $17.22 \pm 28.657$ & $13.33 \pm 20.864$ & 0.821 \\
Week 4 & $10 \pm 19.704$ & $8.24 \pm 14.246$ & 1.000 \\
Week 8 & $2.78 \pm 7.519$ & $9.41 \pm 17.843$ & 0.291 \\
Electric shock-like pain & & & \\
Baseline (Week 0) & $22.22 \pm 30.785$ & $21.11 \pm 23.736$ & 0.973 \\
Week 4 & $13.89 \pm 23.044$ & $9.41 \pm 17.489$ & 0.545 \\
Week 8 & $5.56 \pm 11.490$ & $6.47 \pm 14.552$ & 0.637 \\
Tingling sensation & & & 0.055 \\
Baseline (Week 0) & $43.89 \pm 30.705$ & $25.56 \pm 28.537$ & 0.217 \\
Week 4 & $31.67 \pm 31.296$ & $17.65 \pm 20.775$ & 0.105 \\
Week 8 & $27.22 \pm 29.666$ & $12.35 \pm 21.074$ & \\
Numbness & & & 0.737 \\
Baseline (Week 0) & $41.67 \pm 25.952$ & $43.89 \pm 25.699$ & 0.776 \\
Week 4 & $32.22 \pm 27.559$ & $30.59 \pm 26.568$ & 0.867 \\
Week 8 & $27.78 \pm 28.192$ & $24.71 \pm 25.524$ & \\
\hline Data presented as p, & & & \\
\hline
\end{tabular}

Data presented as n; p, significance between experimental and control group; Independent T-test for normally distributed data, Mann-Whitney test for abnormally distributed data. The statistically significant difference is shown as $*(p<0.05)$.

treatment of $100 \mathrm{mg}$ gabapentin and $25 \mathrm{mg}$ amitriptyline were prescribed to 1 subject $(5.6 \%)$ in the control group, and a combined treatment of $300 \mathrm{mg}$ gabapentin and 25 $\mathrm{mg}$ amitriptyline was given to 1 subject $(5.6 \%)$ in the experimental group. However, we found out that there is no difference in standard treatment between both treatment groups $(\mathrm{p}>0.05)$.

\section{Data Analysis}

The effectiveness of treatment can be analyzed through the mean score of the BPI, as displayed in Table 4. First, the BPI score regarding pain with burning sensation recorded the highest mean of 17.22 in the experimental group at week 0. Meanwhile, the lowest mean BPI score was 2.78 , which was found in the experimental group on the $8^{\text {th }}$ week, as seen in Figure 1. Secondly, the highest and lowest mean BPI scores of electric shock-like pain were found in the experimental group, with a score of 22.22 at week 0 and 5.56 at week 8, respectively, and can be found in Figure 2. Next, the highest mean BPI score of a tingling sensation was 43.89 , found in the experimental group at week 0, while the lowest mean BPI score was 12.35 , found in the control group on the $8^{\text {th }}$ week that can be seen in Figure 3. Lastly, the highest and lowest mean BPI score of numbness was found in the control group at week 0 and week 8 , with a score of 43.89 and 24.71, respectively, as seen in Figure 4. The data analysis concluded that the BPI scores of the two treatment groups did not differ significantly each week $(\mathrm{p}>0.05$.) 


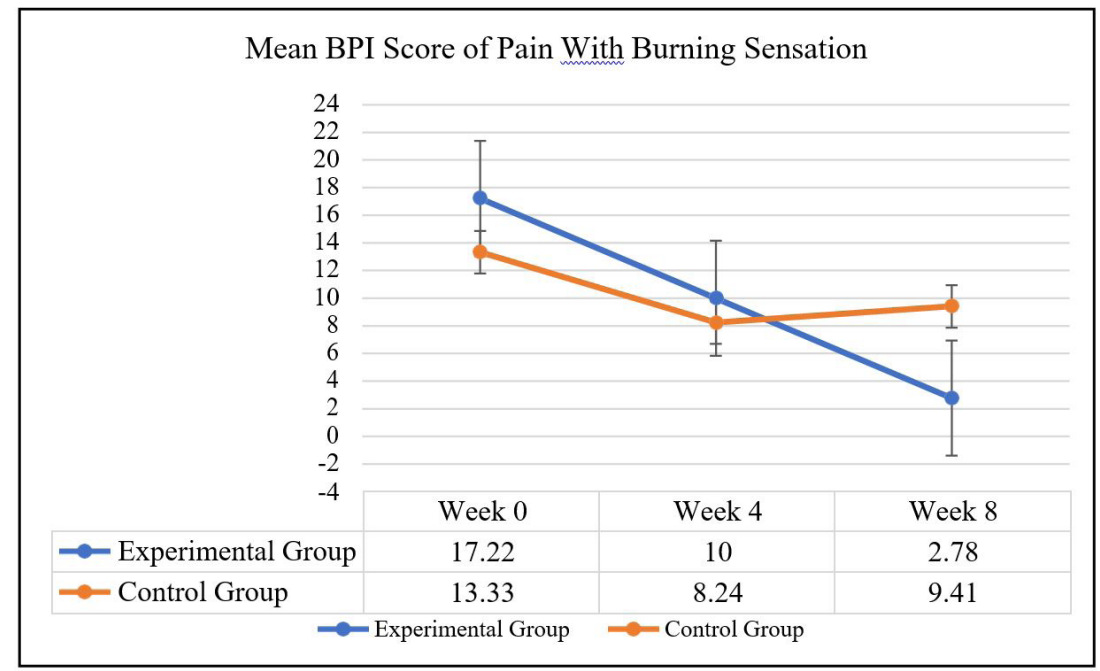

Figure 1. Mean BPI score of pain with burning sensation of experimental group and control group

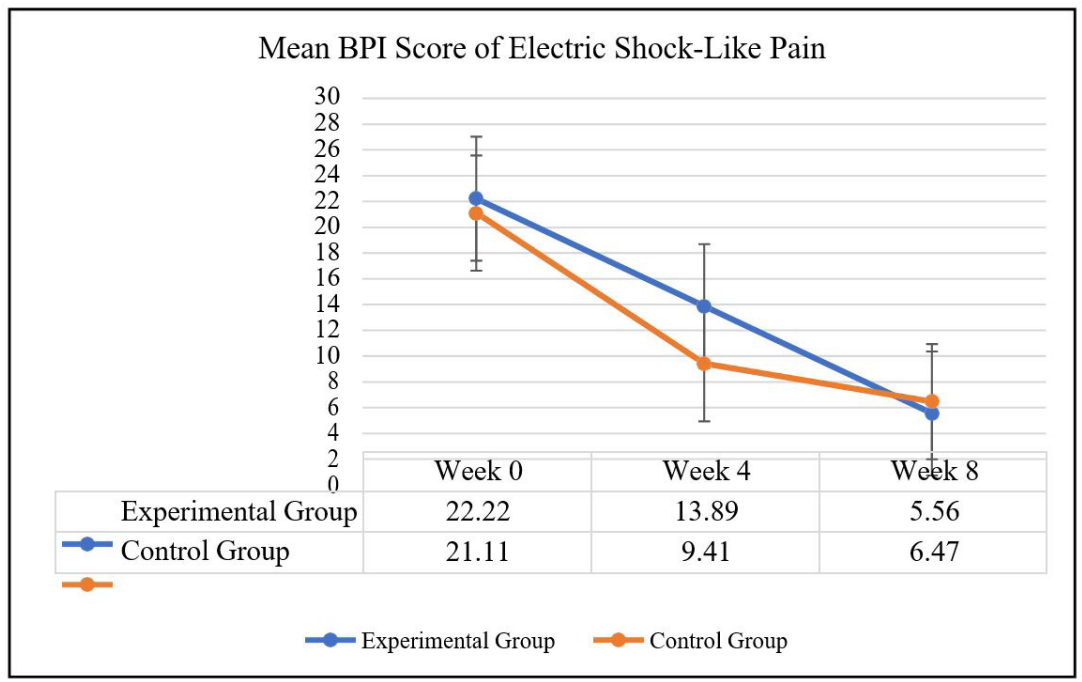

Figure 2. Mean BPI Score of electric shock-like pain of experimental group and control group

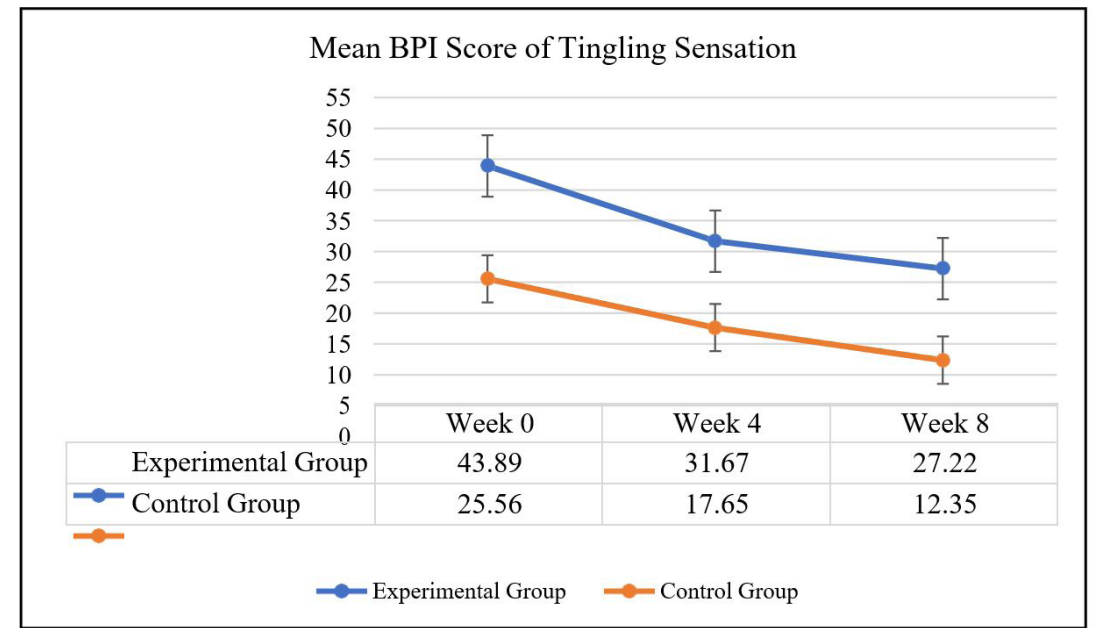

Figure 3. Mean BPI score of pain with tingling sensation of experimental group and control group 


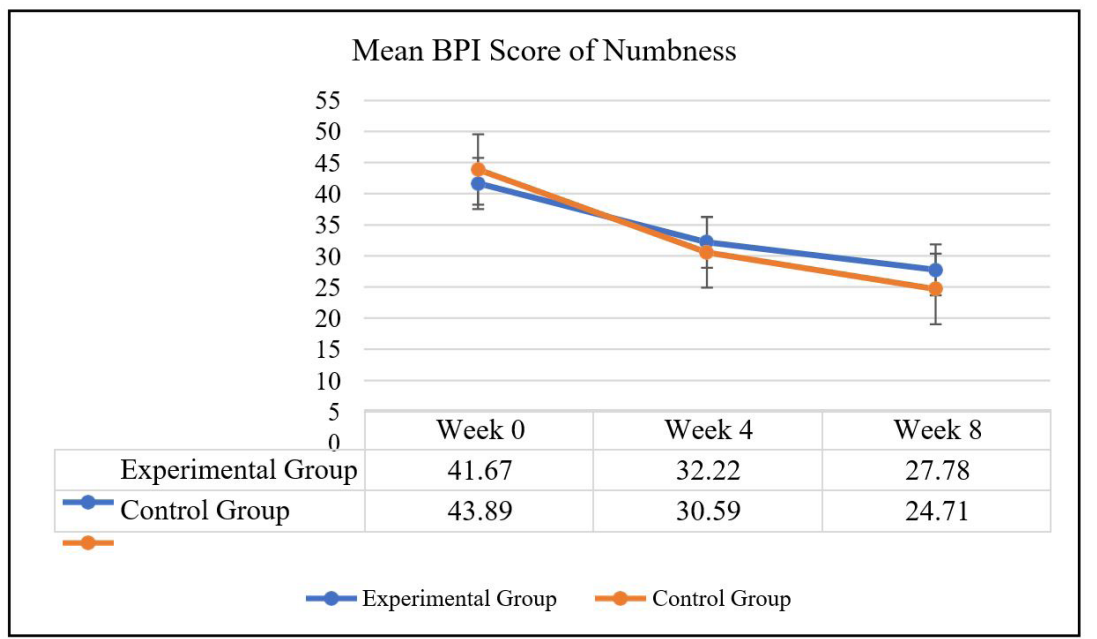

Figure 4. Mean BPI score of numbness of experimental group and control group

Table 5. Mean difference of BPI score between experimental group and control group

\begin{tabular}{llll}
\hline BPI Score & $\begin{array}{l}\text { Experimental Group } \\
(\mathbf{n}=\mathbf{1 8})\end{array}$ & Control Group (n=17) & $\boldsymbol{p}$ \\
\hline Pain with burning sensation & & & \\
$\Delta$ BPI score week 0-4 & $7.222 \pm 18.408$ & $5.882 \pm 13.256$ & $0.021^{*}$ \\
$\Delta$ BPI score week 4-8 & $7.222 \pm 18.725$ & $-1.176 \pm 13.173$ & 0.3040 \\
$\Delta$ BPI score week 0-8 & $14.444 \pm 27.272$ & $4.705 \pm 21.247$ & $0.035^{*}$ \\
Electric shock-like pain & & & \\
$\Delta$ BPI score week 0-4 & $8.333 \pm 16.179$ & $11.176 \pm 19.326$ & $0.002^{*}$ \\
$\Delta$ BPI score week 4-8 & $8.333 \pm 18.230$ & $2.941 \pm 5.878$ & $0.007^{*}$ \\
$\Delta$ BPI score week 0-8 & $16.667 \pm 26.121$ & $14.117 \pm 20.934$ & $0.001^{*}$ \\
Tingling sensation & & & \\
$\Delta$ BPI score week 0-4 & $12.222 \pm 16.647$ & $9.411 \pm 14.348$ & $0.000^{*}$ \\
$\Delta$ BPI score week 4-8 & $4.444 \pm 9.217$ & $5.294 \pm 13.284$ & $0.010^{*}$ \\
$\Delta$ BPI score week 0-8 & $16.667 \pm 18.786$ & $14.705 \pm 24.268$ & $0.000^{*}$ \\
Numbness & & & \\
$\Delta$ BPI score week 0-4 & $9.444 \pm 9.375$ & $11.764 \pm 13.339$ & $0.000^{*}$ \\
$\Delta$ BPI score week 4-8 & $4.444 \pm 6.156$ & $5.882 \pm 12.776$ & $0.006^{*}$ \\
$\Delta$ BPI score week 0-8 & $13.888 \pm 11.447$ & $17.647 \pm 22.508$ & $0.000^{*}$ \\
\hline
\end{tabular}

Data presented as n; p, significance between experimental and control group; Independent T-test for normally distributed data, Mann-Whitney test for abnormally distributed data. The statistically significant difference is shown as $*(p<0.05)$.

The results of data analysis using the Wilcoxon test showed that the most significant mean difference for each symptom could be seen on the BPI score of week 0 to week 8 , as displayed in Table 5. For that particular period, the BPI score of pain with a burning sensation in the experimental group showed the most remarkable mean difference of 14.445, while for the control group, it was 4.705 with a p-value of 0.035 . Meanwhile, the mean difference of BPI score of electric shock-like pain was 16.667 in the experimental group and 14.117 in the control group with a p-value of 0.001 . Simultaneously, for the tingling sensation symptom, the mean difference of the BPI score was 16.667 in the experimental group and 14.705 in the control group with a p-value of 0.010 . While for numbness, the mean difference of the BPI score was 13.888 and 17.647 in the experimental group and the control groups, respectively (p-value of 0.000 ).

The improvement of the mean difference of BPI score in the impact of pain such as pain with burning sensation, electric shock-like pain, and the tingling sensation from week 0 to week 8 was found to be greater in the experimental group compared to the control group. Meanwhile, the improvement of the mean difference of 
BPI score of numbness from week 0 to week 8 was higher in the control group compared to the experimental group. The results indicated that treatment in patients, for both symptomatic only and combined treatment of astaxanthin with symptomatic treatment, can significantly enhance the pain relief in patients' daily activities $(p<0.05)$.

Both treatments have been proven to significantly reduce the severity of pain's impact on the patients' daily activities. The impact of pain or symptoms felt by patients, such as pain with a burning sensation, electric shock-like pain, numbness, and tingling sensation, showed different improvements. The differences might be due to different mechanisms of the pathophysiological process of neuropathic pain ("individualized neuropathic pain based on phenotyping") and genetic factors.

The most significant improvement was observed in the improvement of numbness symptoms in the control group from week 0 to week 8 , with the value of 17.647 \pm 22.508 . The second greatest improvement was found in the improvement of electric shock-like pain in the experimental group from week 0 to week 8, which accounted for $16.667 \pm 26.121$, as well as a tingling sensation in the experimental group from week 0 to week 8 , with a range of $16.667 \pm 18.786$. The data showed that the addition of astaxanthin adjunctive therapy in the standard treatment of symptomatic treatment is better than symptomatic treatment alone in improving pain relief in patients.

\section{DISCUSSION}

The Role of Astaxanthin in improving The Impact of Pain in Painful Diabetic Neuropathy Patients

Painful diabetic neuropathy in patients with diabetes mellitus is caused by a disruption in the vascular and metabolic systems, resulting in hyperglycemia conditions which eventually lead to the increased production of the aldose reductase enzyme. The increase of this enzyme production can further lead to a buildup of sorbitol and polyols, leading to overproduction of oxidative stress, increases in (advanced glycation end products (AGEs), and decreases in nerve growth factor (NGF), consequently causing damage and inflammation to nerve cells.

Astaxanthin is a fat-soluble xanthophyll carotenoid that plays an essential role in treating painful diabetic neuropathy and other diabetes complications. The antioxidant, anti-inflammatory, and anti-diabetic properties of astaxanthin can improve pain relief in patients' daily activities. Astaxanthin is neuroprotective (Lin, Wen, \& Tsai, 2018), which means that it will protect the nervous system against further damage due to diabetes complications through the inhibition of inflammatory response via nuclear factor kappa-lightchain-enhancer of activated B cells (NF- $\kappa \mathrm{B}$ ) pathway, the inhibition of microvascular damage by increasing vascular endothelial growth factor (VEGF) production, and the inhibition of apoptosis through the regulation of a mitogen-activated protein kinase (MAPK) and PI3K/Akt pathways. Research conducted by Lin, et al., (2018) proved that astaxanthin has neuroprotective properties with $\mathrm{p}<0.05$. Another study which resulted in a $p$-value of $\leq 0.0001$, also successfully proved that adjunctive therapy of astaxanthin reduces the pain felt by patients, repairs metabolic processes or blood supply to the nervous system, and improves other symptoms of diabetic neuropathy.

Furthermore, astaxanthin adjunctive therapy on standard treatment can increase endogenous antioxidants, glutathione, and NGF. It can decrease AGEs and malondialdehyde levels. These processes will reduce oxidative stress and inhibit inflammatory biomarkers, such as interleukin (IL)-1 $\beta$, IL-6, and tumor necrosis factor-alpha (TNF- $\alpha$ ). Reduced oxidative stress and inhibited inflammatory biomarkers can give rise to insulin production, and decrease insulin resistance and blood sugar levels. In addition, astaxanthin can also inhibit the production of inflammation mediators by suppressing the activation of cyclooxygenase-2 (COX2) and inducible nitric oxide synthase (iNOS) biomarker protein degradation, which also lead to the suppression of oxidative stress, cell apoptosis, and the suppression of chronic neuroinflammation caused by diabetes. Thus, making astaxanthin a multitargeted therapy.

Interestingly, our results found out that the control and experimental groups significantly reduced the impact of pain $(p<0.05)$. However, the average weekly BPI score did not exhibit a significant difference $(p>0.05)$. This could be explained due to the abnormal distribution of data, extensive range of data, and the power of the study, which was $80 \%$. Power of study is the ability of research to show significant differences so that a difference of less than $20 \%$ will not appear to be significantly different.

\section{Side Effect}

Nevertheless, we discovered that a staxanthin adjunctive therapy in standard treatment causes a side effect in 1 subject from the experimental group in the form of itching skin, as shown in Table 6 . The symptoms experienced by the patient are slightly mild and did not lead to severe complications. Hence, the condition did not require any further treatment. A study conducted by Davinelli, Scapagnini, \& Nielsen (2018), also reported similar conditions, where the administration of astaxanthin to research subjects caused side effects such as pruritus. Therefore, the side effect might be affected by this study's limitations, which was the lack of information about the 
Table 6. Side effect of treatment in experimental group and control group

\begin{tabular}{llll}
\hline & $\begin{array}{l}\text { Experimental Group } \\
(\mathbf{n}=\mathbf{1 8})\end{array}$ & $\begin{array}{l}\text { Control Group } \\
(\mathbf{n}=\mathbf{1 7})\end{array}$ & $\boldsymbol{p}$ \\
\hline Side Effect of Treatment & & & \\
Yes & $1(5.6)$ & $0(0)$ & 1.00 \\
No & $17(94.4)$ & $17(100)$ & \\
\hline
\end{tabular}

Data presented as n; p, significance between experimental and control group; Independent T-test for normally distributed data, Mann-Whitney test for abnormally distributed data, Wilcoxon test for comparing the significance between a pair of data. The statistically significant difference is shown as $*(\mathrm{p}<0.05)$.

patient's past medical history, more importantly about the patient's diabetes and allergies history.

\section{CONCLUSION}

In comparison to patients who were only given a standard treatment such as gabapentin, pregabalin, or amitriptyline for diabetic neuropathic pain, additional therapy of astaxanthin at a dose of $6 \mathrm{mg}$ once per day was found to have significantly improved the BPI in patients that have been diagnosed with painful diabetic neuropathy, as seen in the experimental group.

\section{ACKNOWLEDGMENT}

Authors have no acknowledgments to declare.

\section{CONFLICT OF INTEREST}

Authors have no conflict of interest to declare.

\section{REFERENCES}

American Diabetes Association. (2014). Diagnosis and Classification of Diabetes Mellitus. Diabetes Care.

Cavan David, Fernandes, J., Makaroff, L., Ogurtsova, K., \& Webber, S. (2015). IDF Diabetes Atlas 7th ed. International Diabetes Federation.

CDC. (2020). Diabetes and Your Heart. Centers for Disease Control and Prevention.

Chang, M., \& Xiong , F. (2020). Astaxanthin and its Effects in Inflammatory Responses and InflammationAssociated Diseases: Recent Advances and Future Directions. MDPI, 25.

Davinelli, S., Scapagnini, G., \& Nielsen, M. (2018). Astaxanthin in Skin Health, Repair, and Disease: A Comprehensive Review. MDPI.

Forstenpointner, J., Otto, J., \& Baron, R. (2018). Individualized neuropathic pain therapy based on phenotyping: are we there yet? The Journal of the
International Association for the Study of Pain, 159(3), 569-575.

Jankovic, M., Novakovic, I., Nikolic, D., Maksic, J., Petronic, I., \& Cirovic, D. (2021). Genetic and Epigenomic Modifiers of Diabetic Neuropathy. International Journal of Molecular Sciences.

Javed, S., Petropoulos, I., Alam, U., \& Malik, R. (2015). Treatment of painful diabetic neuropathy. Therapeutic Advances in Chronic Disease.

Kaur, S., \& Pandhi, P. (2011). Painful diabetic neuropathy: an update. Annals of Neurosciences.

Kharroubi, A., \& Darwish, H. (2015). Diabetes mellitus: The epidemic of the century. World Journal of DIabetes.

Lin, W.-N., Wen, Y.-T., \& Tsai, R.-K. (2018). Neuroprotective effect of astaxanthin in a rat model of anterior ischemic optic neuropathy. Investigative Ophthalmology \& Visual Science.

Ministry of Health of the Republic of Indonesia. (2018). Hari Diabetes Sedunia Tahun 2018. Pusat Data dan Informasi Kementerian Kesehatan RI.

Papatheodorou, K. (2018). Complications of Diabetes 2017. Journal of Diabetes Research.

Petrie, J., Guzik, T., \& Touyz, R. (2018). Diabetes, Hypertension, and Cardiovascular Disease: Clinical Insights and Vascular Mechanisms. The Canadian Journal of Cardiology.

Raputova, J., Srotova, I., Vlckova, E., Sommer, C., Üçeyler, N., \& Birklein, F. (2017). Sensory phenotype and risk factors for painful diabetic neuropathy: a cross-sectional observational study. The Journal of the International Association for the Study of Pain, 158(12), 2340-2353.

Singh, J., \& Rajbhandari, S. (2014). Pathogenesis of Painful Diabetic Neuropathy. Hindawi Pain Research and Treatment, 2014. 
Snyder, M., Gibbs, L., \& Lindsay, T. (2016). Treating Painful Diabetic Peripheral Neuropathy: An Update. American Family Physician.

Talukdar, J., Bhadra, B., Dattaroy, T., Nagle, V., \& Dasgupta, S. (2020). Potential of natural astaxanthin in alleviating the risk of cytokine storm in COVID-19. Elsevier Public Health Emergency Collection.
Wafa, A., El-Nahas, M., Al-Biaumy, A., \& Mansour, Y. (2017). Study of advanced glycation endproducts and their receptors in Egyptian type 2 diabetic individuals with peripheral neuropathy. Egyptian Journal of Obesity, Diabetes, and Endocrinology, 3(1), 15-21.

Wisse, B. (2018). Long-term complications of diabetes. U.S. National Library of Medicine. 